EDITORIAL

\title{
How good is anticoagulation control in non-valvar atrial fibrillation? Observations on the elderly, ethnicity, patient perceptions, and understanding of AF thromboprophylaxis
}

\section{A Choudhury, G Y H Lip}

Heart 2005;91:425-426. doi: 10.1136/hrt.2004.052480

There continues to be a wide variation of opinion among UK consultants regarding the best treatment strategies for ensuring effective thromboprophylaxis of atrial fibrillation

\begin{abstract}
trial fibrillation (AF) is the most common encountered cardiac rhythm disturbance in clinical practice, with its prevalence increasing from $1 \%$ in those under the 50 years to around $10 \%$ in those over 80 years. $^{1}$ Importantly, AF is an independent predictor of stroke, with an annual risk that is 5-6 times higher than patients in sinus rhythm. However, this risk is not homogeneous, ranging from an annual risk of $1 \%$ in patients aged $<65$ years old with no risk factors, to over $12 \%$ per year in patients who have a history of prior stroke or transient ischaemic attack. ${ }^{2}$ Not only is AF a major risk factor for stroke, when strokes do occur in association with AF, the patients suffer a substantial mortality, morbidity, disability, and longer hospital stay. With the aging population, the burden of $\mathrm{AF}$ is expected to double in the next two generations, making this arrhythmia an increasingly important public health issue. ${ }^{2}{ }^{3}$
\end{abstract}

\section{THROMBOPROPHYLAXIS}

The benefits of thromboprophylaxis in patients with AF are well established, ${ }^{2}$ and most guidelines recommend the use of anticoagulation with warfarin for high risk patients. However, the dose-response of warfarin is complex and its activity is easily altered by concurrent medications, food interactions, alcohol, and illnesses. Adherence to medical advice and routine monitoring of the international normalised ratio (INR) is important, and in many countries (including the UK), the service is usually provided by specialised anticoagulant clinics-although some general practitioners can offer point-of-care testing. However, there continues to be wide variation in management (and disagreement) among UK consultants regarding the best treatment strategies. ${ }^{4}$ What is also apparent is that many patients with AF possess very limited knowledge of AF as well as its consequences and treatment. ${ }^{6}$ These points have important implications regarding effective anticoagulation control for thromboprophylaxis of AF.

Despite clear guidelines on risk stratification for stroke in non-valvar $\mathrm{AF}$ patients and the appropriateness of oral anticoagulation in high risk patients, ${ }^{2}$ repeated surveys have shown that only $20-30 \%$ of AF patients are on warfarin, although if the guidelines are adhered to, around $50-60 \%$ of them should probably be anticoagulated. ${ }^{7}$ Elderly patients (arbitrarily defined as age $>75$ years) are often suboptimally treated, despite this age group being most likely to benefit most from anticoagulation, in view of the high absolute risk of stroke in such patients. Indeed, the decision to anticoagulate an elderly patient is often (supposedly) individualised, depending on underlying co-morbidities, concomitant drug therapy, and physician (and/or patient, if given the scope) preferences.

Nonetheless, physician perceptions of riskbenefit are not always reliable, and bleeding risks are often overestimated..$^{5}$ One questionnaire survey revealed that only $52 \%$ of geriatricians and $40 \%$ of general physicians viewed lone $\mathrm{AF}$ in patients above 75 years as an indication for anticoagulation, although $94 \%$ of the same physicians agreed that the above patient group were at a higher risk of stroke than younger patients. ${ }^{8}$ The paradox is that many patients under the age of 65 with lone AF (who are at the lowest risk of embolic events) are often considered for anticoagulation, while the use of warfarin in those aged $>75$ with lone AF who are often at a high risk of such events remains disappointing.

While disability, cognitive impairment, and problems with compliance are common in the elderly patients with $\mathrm{AF}$, they are not substantially different from the elderly population in general and can often be overcome. ${ }^{9}$ Indeed, many elderly patients would accept treatment when the risks and benefits of warfarin treatment are properly explained. ${ }^{9}$ Despite such counselling, we recognise that some will still decline treatment with warfarin for a wide variety of patient related reasons - that is, "informed dissent" ${ }^{\prime 10}$-including the inconvenience of dosing adjustments and regular blood tests to monitor INR values, dietary restrictions, the risk of minor and major bleeding, and under-appreciation or lack of knowledge regarding the risk of stroke, or poor adherence to the treatment regimen. However, recent studies have suggested that dependency and associated cognitive and functional impairment-rather than age itself-increases the risk of warfarin related bleeding in the elderly. ${ }^{11}{ }^{12}$

\section{THE PATIENT PERSPECTIVE}

Of more concern is that many patients with AF possess very limited knowledge of AF as well as its consequences and treatment, and that only a minority felt that their doctor had given them 
"enough information" about their warfarin therapy. ${ }^{6}$ One study of AF patients attending a teaching hospital anticoagulant clinic reported that only $52 \%$ of the patients were aware of the reason(s) for commencing warfarin, while $37 \%$ were unaware of the risks of stroke and thrombosis with AF; furthermore, only $45 \%$ of the cohort knew that there was some associated risk of "bleeding" or "poisoning" with warfarin. Most patients were taking warfarin because "their doctor told them to".

Furthermore, there are also major ethnic differences in the patients' perceptions of AF and anticoagulation, with IndoAsians and Afro-Caribbeans being significantly less aware of AF compared to their white counterparts. ${ }^{6}$ Interestingly, most Indo-Asians feel that the control of their health is with God or "fate", whereas most Afro-Caribbeans felt that the control was with them individually; in contrast, white Europeans considered that control of their health was with them or their doctors. ${ }^{6}{ }^{13}$ Indeed, ethnicity is an important factor in deciding adherence to medical treatment, especially when there are language barriers. ${ }^{13}{ }^{14}$

\section{OPTIMISING THROMBOPROPHYLAXIS FOR AF}

How do these issues translate to our management of anticoagulation for AF? In this issue of Heart, Jones and colleagues $^{15}$ report that AF patients treated with warfarin spent $32.1 \%$ of the time outside the target INR range, and a $10 \%$ increase in the time spent outside the range was associated with an increased risk of mortality and of an ischaemic stroke and other thromboembolic events. This would broadly be in keeping with the findings of Bungard and colleagues, ${ }^{16}$ who found that on hospitalisation only one third of AF patients were found to have their INR in the therapeutic range, and those admitted with any thromboembolic event or ischaemic stroke were significantly more likely to have subtherapeutic INRs.

Thus, warfarin may well be underprescribed in AF patients, but these studies show that even treated patients are often under- or over-anticoagulated, resulting in either inadequate protection from thromboembolic events or increased risk of bleeding. Given the complexity of the pharmacokinetic and pharmacodynamic profile of warfarin and the lack of patient education and understanding of the drug and their underlying illness, maintaining the INR within the desired range for the majority of the time can be difficult, especially since the risk of ischaemic events and haemorrhagic strokes rises with under- and over-anticoagulation, respectively, and may offset the potential benefits of warfarin therapy.

How can things improve? There is good evidence that adherence to medical treatment is enhanced by knowledge and understanding of the underlying condition and the relevant treatment with its benefits and side effects. ${ }^{17}$ Indeed, $\mathrm{AF}$ is one such condition in which active patient involvement in treatment decisions may substantially impact management, and shared decision making may improve health outcomes. ${ }^{18}{ }^{19}$ Vulnerable groups highlighted earlier, such as the elderly and ethnic minorities, may require additional attention to improve AF thromboprophylaxis.

However, in the brief, hurried encounters that typify outpatient practice, we are rarely able to provide the quantity and quality of information required for patients to participate in shared decision making. ${ }^{20}$ Nevertheless, an understanding of how patients feel about warfarin therapy is important, as this may be a potential reason for non-compliance. Patients' understanding (and hence, compliance) can be potentially improved by encouraging them to ask questions, by providing them with leaflets in their own language, having them meet specialist nurses, or by using decision aids (for example, audio booklets and interactive video discs). The latter have been shown to improve patient knowledge and comfort with a variety of conditions, including $\mathrm{AF}$, as well as stimulate their participation in decision making without increasing anxiety. ${ }^{21}$ The study by Gage and colleagues $^{18}$ found that antithrombotic treatment based on patient preferences was more cost effective in terms of quality adjusted life-years of survival, and improving patients' knowledge and satisfaction significantly improves patient compliance.

The study by Jones and colleagues ${ }^{15}$ highlights difficulties with anticoagulation control and important deficiencies in our provision of thromboprophylaxis to AF patients. This is all the more timely, with the planned guidelines on atrial fibrillation risk assessment, diagnosis, treatment, and review (www.nice.org.uk) and the availability of new antithrombotic drugs on the horizon that may do away with the need for anticoagulation monitoring. Maybe, things can only get better!

\section{Authors' affiliations}

A Choudhury, G Y H Lip, Haemostasis Thrombosis and Vascular Biology Unit, University Department of Medicine, City Hospital, Birmingham, UK

Competing interests: GYHL is Clinical Adviser to the Guideline Development Group developing the NICE Guidelines on Atrial Fibrillation. In addition, he has received research funding and honoraria for meetings, educational symposia and advisory boards from various pharmaceutical companies involved in antithrombotic agents and other drugs used in the management of atrial fibrillation.

\section{REFERENCES}

1 Freestone B, Lip GYH. Epidemiology and costs of cardiac arrhythmias. In: Lip GYH, Godffredsen J, eds. Cardiac arrhythmias: a clinical approach. Edinburgh: Mosby, 2003:3-24.

2 Lip GYH, Hart RG, Conway D. ABC of antithrombotic therapy: antithrombotic therapy for atrial fibrillation. BMJ 2002;325:1022-5.

3 Wattigney WA, Mensah GA, Croft JB. Increasing trends in hospitalization for atrial fibrillation in the United States, 1985 through 1999: implications for primary prevention. Circulation 2003;108:711-16.

4 Williams E, Ansari M, Lip GYH. Managing atrial fibrillation in the accident and emergency department. QJM 2001;94:609-14.

5 Lip GYH, Zarifis J, Watson RDS, et al. Physician variation in the management of patients with atrial fibrillation. Heart 1996;75:200-5.

6 Lip GYH, Kamath S, Jafri M, et al. Ethnic differences in patient perceptions of atrial fibrillation and anticoagulation therapy: the West Birmingham atrial fibrillation project. Stroke 2002;33:238-42.

7 Sudlow M, Thomson R, Thwaites B, et al. Prevalence of atrial fibrillation and eligibility for anticoagulants in the community. Lancet 1998;352:1167-71.

8 Vasishta S, Toor F, Johansen A, et al. Stroke prevention in atrial fibrillation: physicians' attitudes to anticoagulation in older people. Arch Gerontol Geriatr $2001 ; 33: 219-26$.

9 Sudlow M, Thomson R, Kenny RA, et al. A community survey of patients with atrial fibrillation: associated disabilities and treatment preferences. $\mathrm{Br} J \mathrm{Gen}$ Pract 1998;48: 1775-8.

10 Howitt A, Armstrong D. Implementing evidence-based medicine in general practice: audit and qualitative study of antithrombotic treatment for atrial fibrillation. BMJ 1999;318:1324-7.

11 Lackie CL, Garbarino KA, Pruetz JA. Warfarin therapy for atrial fibrillation in the elderly. Ann Pharmacother 2002;36:200-4.

12 Goudie BM, Donnan PT, Fairfield G, et al. Dependency rather than old age increases the risk of warfarin-related bleeding. Br J Gen Pract 2004;54:690-2.

13 Nadar S, Begum N, Kaur B, et al. Patients' understanding of anticoagulant therapy in a multiethnic population. J R Soc Med 2003;96:175-9.

14 Hellman S, Baker L, Flores D, et al. Effect of ethnicity on adherence to diabetic regimen. Ethnicity Dis 1997;7:221-8.

15 Jones M, McEwan P, Morgan CL, et al. Evaluation of the pattern of treatment, level of anticoagulation control, and outcome of treatment with warfarin in patients with non-valvar atrial fibrillation: a record linkage study in a large British population. Heart 2005;91:472-7.

16 Bungard TJ, Ackman ML, Ho G, et al. Adequacy of anticoagulation in patients with atrial fibrillation coming to a hospital. Pharmacotherapy 2000;20:1060-5.

17 Arnsten JH, Gelfand JM, Singer DE. Determinants of compliance with anticoagulation: a case control study. Am J Med 1997; 103:11-17.

18 Gage BF, Cardinalli AB, Owens DK. Cost-effectiveness of preference-based antithrombotic therapy for patients with nonvalvular atrial fibrillation. Stroke 1998;29:1083-91.

19 Coulter A, Entwistle V, Gilbert D. Sharing decisions with patients: is the information good enough? BMJ 1999;318:318-22.

20 Braddock $\mathrm{CH}$, Edwards KA, Hasenberg NM, et al. Informed decision making in outpatient practice: time to get back to basics. JAMA 1999;282:2313-20.

21 O'Connor AM, Rostom A, Fiset V, et al. Decision aids for patients facing health treatment or screening decisions: systematic review. BMJ 1999;319:751-4 